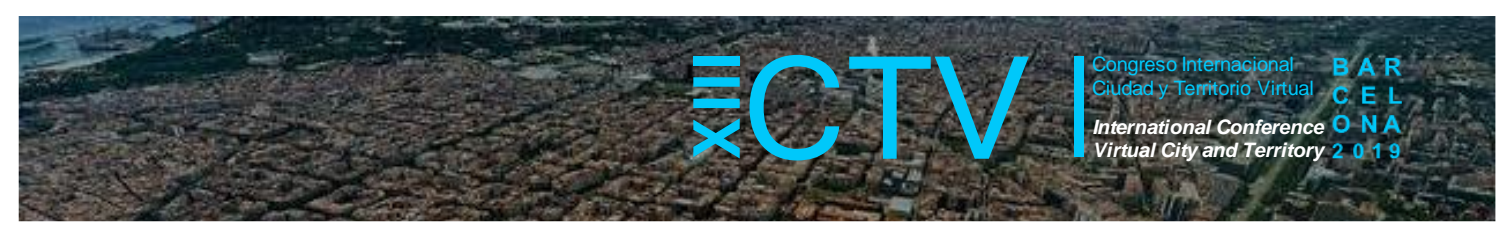

\title{
METODOLOGÍA DE TRABAJO PARA ESTRATEGIAS DE DISEÑO ECOLÓGICO EN CLIMA CÁLIDO HÚMEDO DE MÉXICO
}

\author{
Guzmán-Hernández, Ignacio A. ${ }^{1 *}$; Franco, Fidel $^{2}$ y Roset, Jaime ${ }^{3}$ \\ Remisión inicial: 2019-05-30; Remisión definitiva: 2019-09-18; Publicación: 2019-12-21
}

Citación: Guzmán-Hernández, I. A. et al. (2019). Metodología de trabajo para estrategias de diseño ecológico en clima cálido húmedo de México. En XIII CTV 2019 Proceedings: XIII International Conference on Virtual City and Territory: "Challenges and paradigms of the contemporary city": UPC, Barcelona, October 2-4, 2019. Barcelona: CPSV, 2019, p. 8468. E-ISSN 2604-6512. DOI http://dx.doi.org/10.5821/ctv.8468

\begin{abstract}
Resumen
La necesidad de comodidad de las personas nos ha llevado siempre a modificar nuestro entorno, dentro de dicha búsqueda de comodidad encontramos el confort térmico, de satisfacción mental con el ambiente. En los sitios con clima cálido-húmedo dicho confort térmico es difícil de lograr durante varias temporadas del año, debido a las altas temperaturas que se encuentran acompañadas de altos niveles de humedad, causando sensaciones de bochorno. Este trabajo se enfoca en expresar algunas problemáticas con las que se encuentra la implementación de algunos sistemas de climatización pasiva para proyectos habitacionales con el clima en cuestión, así como unas posibles soluciones que se analizarán. Se expone una metodología sencilla de estrategias de diseño pasivo, o de bajo consumo eléctrico, para disminuir temperaturas y humedades. En el caso de estudio, Villahermosa, Tabasco, solamente se cuentan con condiciones ideales aproximadamente el $4.73 \%$ del tiempo, mientras que los altos niveles de humedad permanecen durante casi todo el año. Las estrategias analizadas en el artículo son: protección solar, ventilación natural y mecánica, geotermia somera pasiva y activa, paneles desecantes y la captación de agua proveniente de la deshumidificación. Se propone la implementación de celdas Peltier en procesos de deshumidificación, que ayuden a condensar el vapor de agua contenido en el aire, para que al ingresar a las viviendas sea más confortable.
\end{abstract}

\begin{abstract}
The need for people's comfort has always led us to modify our environment, within this search for comfort we find thermal comfort, of a state of mental satisfaction with the environment. In places with warm-humid climate, such thermal comfort is difficult to achieve during several seasons of the year, due to the high temperatures that are accompanied by high levels of humidity, causing hot flashes. This essay is focused on expressing some of the problems that appear in the implementation of some passive air conditioning systems for housing projects with the climate in question, as well as some possible solutions to be analyzed. A simple methodology of passive design strategies, or low power consumption, to reduce temperatures and humidity is exposed. In the case study, Villahermosa, Tabasco, only ideal conditions are available approximately $4.73 \%$ of the time, while the high humidity levels remain throughout most of the year. The strategies analyzed in the article are: solar protection, natural and mechanical ventilation, passive and active low enthalpy geothermal, desiccant panels and the collection of water from dehumidification. The implementation of Peltier cells in dehumidification processes is proposed, which help to condense the water vapor contained in the air, so that when entering the homes, it is more comfortable.
\end{abstract}

Palabras Clave: Sistemas pasivos; confort térmico; cálido-húmedo; diseño

Key words: Passive systems; thermal comfort; hot-humid weather, design

\section{Introducción}

Dentro de la arquitectura siempre debemos de considerar diversos factores o necesidades para satisfacer, por un lado, las necesidades estéticas, por otro lado, las necesidades funcionales de las edificaciones y por supuesto, una de las más importantes, las necesidades fisiológicas de los usuarios finales. Dentro de estas encontramos el confort térmico, el cual es definido como "Aquella condición de la mente que expresa satisfacción con el ambiente térmico"(ASHRAE, 2004), quedando completamente dependiente de diversos factores, tanto del usuario, como el

\footnotetext{
1 Departamento de Tecnología de la Arquitectura, UPC, https://orcid.org/0000-0001-6351-754X; ${ }^{2}$ Departamento de física, UPC, https://orcid.org/0000-0002-2579-173X y ${ }^{3}$ Departamento física, UPC, https://orcid.org/0000-0002-05485524. * Correo de contacto: ignacio.alejandro.guzman@upc.edu
} 


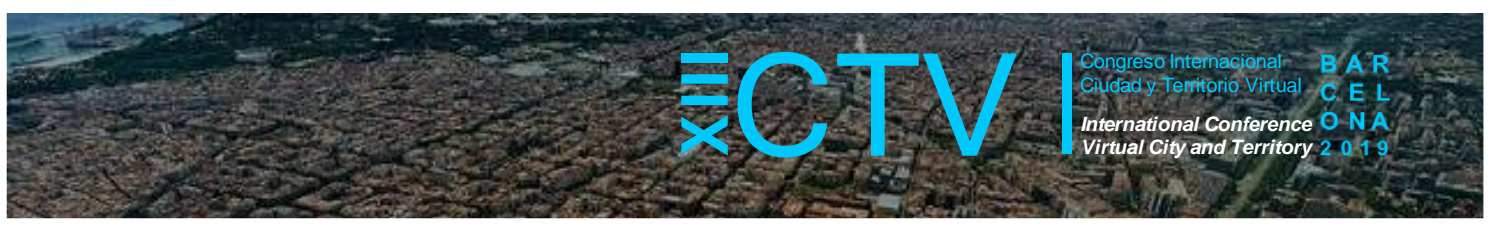

metabolismo, estado de ánimo, adaptabilidad al cambio y el tipo de ropa; como del ambiente; y no solamente refiriéndonos a las condiciones climáticas, sino también a otros elementos físicos del entorno, como la acústica, iluminación y las dimensiones de los espacios.

El confort térmico se ha trabajado exhaustivamente, no solo en la arquitectura, sino también en diversas ingenierías y otras áreas de estudio, logrando establecer en muchos casos parámetros e incluso estándares para lograrlo bajo ciertas condiciones. En la Tabla 1 se muestran algunos de estos parámetros y sus límites, así como sus áreas de aplicación.

Tabla 1. Límites confort térmico

\begin{tabular}{c|c|c|c}
\hline Autor & Temperatura $\left(^{\circ} \mathbf{C}\right)$ & Humedad & Aplicación \\
\hline Givoni & $21-26$ & $5-17 \mathrm{mmHg}$. & General \\
\hline Olgyay & $23.9-29.5$ & $20 \%-75 \%(\mathrm{HR})$ & Trópicos \\
\hline AHSRAE & $21.6-26.6$ & $4-14 \mathrm{mmHg}$. & General \\
\hline Yaglou & $18.8-23.8$ & $30 \%-79 \%(\mathrm{HR})$ & USA \\
\hline ISO 7730 & $20-26$ & $50 \%$ & España \\
\hline
\end{tabular}

Fuente: (Hinz, Gonzalez, De Oteiza, \& Quiros, 1986).

El hecho de que sea un tema ampliamente estudiado, hace que esté en constante evolución con la búsqueda de nuevas tecnologías y estrategias de diseño, en especial para mantener temperaturas óptimas al interior de las edificaciones, puesto que gran parte de la energía utilizada en los edificios es para refrigerar o calentar espacios que de otra manera serían inconvenientes para realizar actividades en ellos. En países como la India, las viviendas emplean aproximadamente el 35\% de la energía para calefacción y refrigeración (Rogers \& Suphachasalai, 2008), en México el promedio es de 18.5\% para el mismo fin (INEGI, 2016) y aunado a eso la mayoría de los países continúa utilizando fuentes no sustentables de energía (R. Yao, Li, \& Liu, 2009). También es importante considerar que muchos de los países con economías en desarrollo están presentando un aumento en el uso de aparatos y consumo energético para lograr confort térmico (Sudhakar, Winderl, \& Priya, 2019) y a su vez provocando mayores gases contaminantes por el alta demanda energética de los edificios (Sharmin, Kabir, \& Rahaman, 2012). En zonas cálido-húmedas son muy empleados los sistemas convencionales de refrigeración, que consumen mucha energía eléctrica, sin embargo, son necesarios debido a que los sistemas pasivos generalmente no son suficientes para proveer condiciones de comodidad a los usuarios, en gran parte debido a las altas temperaturas que normalmente están acompañadas de altos niveles de humedad en el ambiente.

Los seres humanos tenemos formas de deshacernos del calor excedente de la comida que consumimos: Transferencia de calor latente y transferencia de calor sensible; llevadas a cabo por el cuerpo humano y su interacción con el ambiente, por medio de evaporación, conducción, convección y radiación (Choudhury, Majumdar, \& Datta, 2011), el principal problema es que en los sitios con climas cálido-húmedos el aire se encuentra saturado de agua y no permite absorber la transpiración de las personas, haciendo que los cuerpos se mantengan calientes y por ende incómodos. Por esta razón es que el control de la humedad se convierte en unos de los principales problemas a resolver en este tipo de sitios. Los altos niveles de humedad también traen varios problemas, aparte del confort; se ha demostrado que a niveles superiores del $70 \%$ de humedad relativa en el interior de las edificaciones, prolifera la reproducción de microrganismos, como ácaros, hongos, protozoarios, bacterias y virus (Arundel, Sterling, 


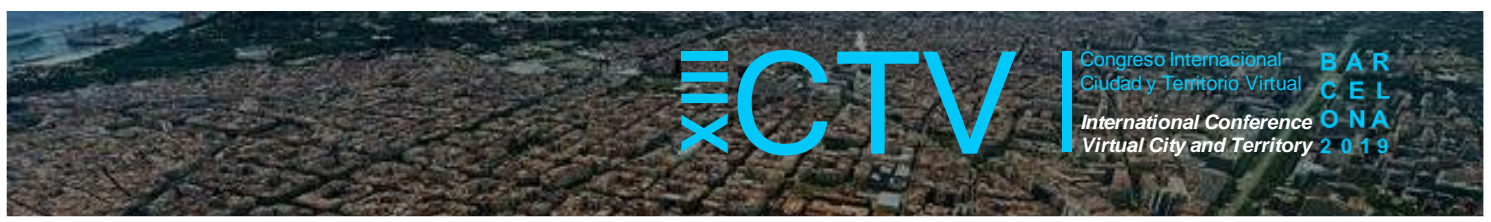

Biggin, \& Sterling, 1986), aumentando el riesgo de contraer o empeorar enfermedades respiratorias como el asma (Andersen \& Korsgaard, 1986).

Es posible que se continúen utilizando equipos de aire acondicionado y deshumidificadores en estas regiones, sin embargo, reducir su consumo lo más que se puede debe de ser una de nuestras prioridades como arquitectos. Un estudio realizado en varias zonas de Chile analizó la ventilación como método único para reducir el consumo energético de viviendas unifamiliares, logrando reducir hasta un $30 \%$ en sitios húmedos con temperaturas mayores a los $30^{\circ}$ (Palme, Aldunate, \& Huerta, 2016).

\subsection{Caso de estudio - Villahermosa, Tabasco, México}

México es el décimo tercer país en extensión, con 1,964,275 km², que junto con su característica topografía hacen que tenga una gran diversidad climática, agrupados en 4 grupos: Clima seco, clima templado, clima cálido semi-húmedo y clima cálido húmedo, cada uno con sus sub-tipos e incluso microclimas por regiones. La ciudad de Villahermosa, capital del estado de Tabasco, se encuentra en el cuarto de los principales grupos como se observa en la figura 1, presentando lluvias todo el año. Villahermosa se encuentra en la latitud norte 17059', longitud oeste $92^{\circ} 56^{\prime}$ y una altitud promedio de 9 m.s.n.m. y el total de las precipitaciones anuales rondan los $2500 \mathrm{~mm}$ (Cervantes et al., 2000). La temperatura promedio en la zona es de $27^{\circ} \mathrm{C}$, sin embargo, la máxima suele alcanzar los $41^{\circ} \mathrm{C}$ y la mínima tiene un promedio de $17.4{ }^{\circ} \mathrm{C}$, mientras que la humedad relativa se encuentra entre un $69 \%$ y un $86.5 \%$, manteniendo un promedio del $80 \%$. La velocidad del viento promedio es de $9.95 \mathrm{~km} / \mathrm{h}$, que es equivalente a $2.76 \mathrm{~m} / \mathrm{s}$.

Figura 1. Mapa de zonas climáticas del sureste mexicano

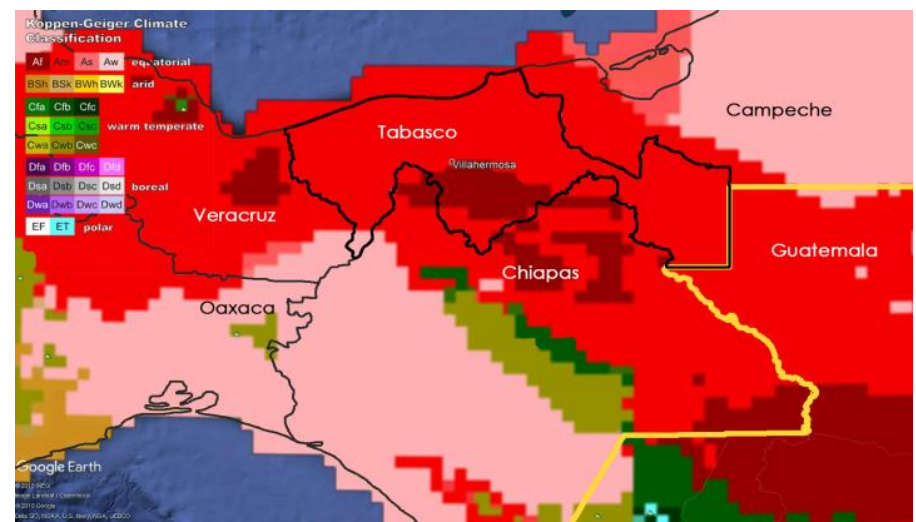

Fuente: World map of the Köppen-Geiger climate classification (Kottek, Grieser, Beck, Rudolf, \& Rubel, 2006).

Estas condiciones climatológicas hacen que al compararlas con los estándares propuestos para el confort térmico se encuentren la mayor parte del tiempo fuera de dichos límites, haciendo que los usuarios de las edificaciones tengan que recurrir a sistemas activos para poder realizar casi todas las actividades. Por todo esto la ciudad de Villahermosa, Tabasco, es un sitio idóneo como caso de estudio para estudiar posibles soluciones pasivas y activas que ayuden a lograr condiciones de confort térmico. 


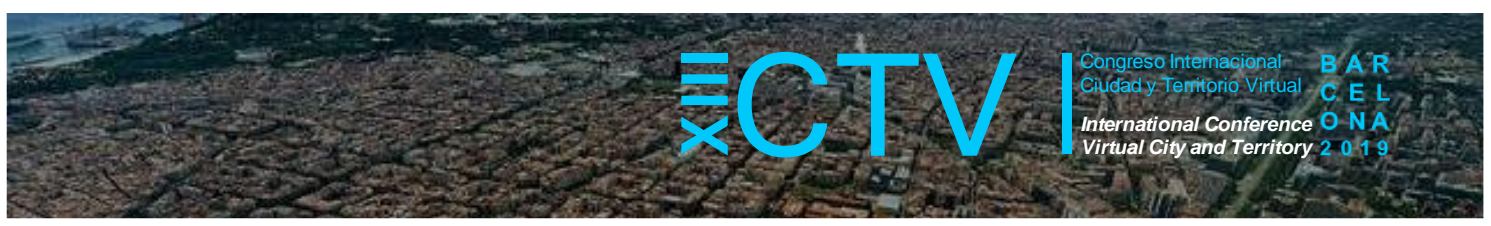

\section{Desarrollo}

En México no existe hasta el momento un estándar para confort térmico, por lo cual es necesario tomar casos análogos con condiciones similares al caso de estudio para poder evaluarlo de manera correcta. Uno de los autores que ha propuesto parámetros de confort para regiones tropicales es Víctor Olgyay, quien después de realizar varios análisis propuso que la temperatura debe de mantenerse entre $23.3 \stackrel{\circ}{\circ} \mathrm{C}$ y $26.7^{\circ} \mathrm{C}$, mientras que la humedad relativa no debe de bajar de un $30 \%$, ni superar el $70 \%$ para que los usuarios se sientan cómodos con el medio (Olgyay \& Frontado, 1998). Con estos parámetros se analizaron los datos climatológicos recopilados en Villahermosa y se plasmaron en un ábaco psiclométrico y como se puede observar en la figura 2 las condiciones del ambiente no favorecen casi nunca al confort climático.

Figura 2. Diagrama psiclométrico con datos de Villahermosa

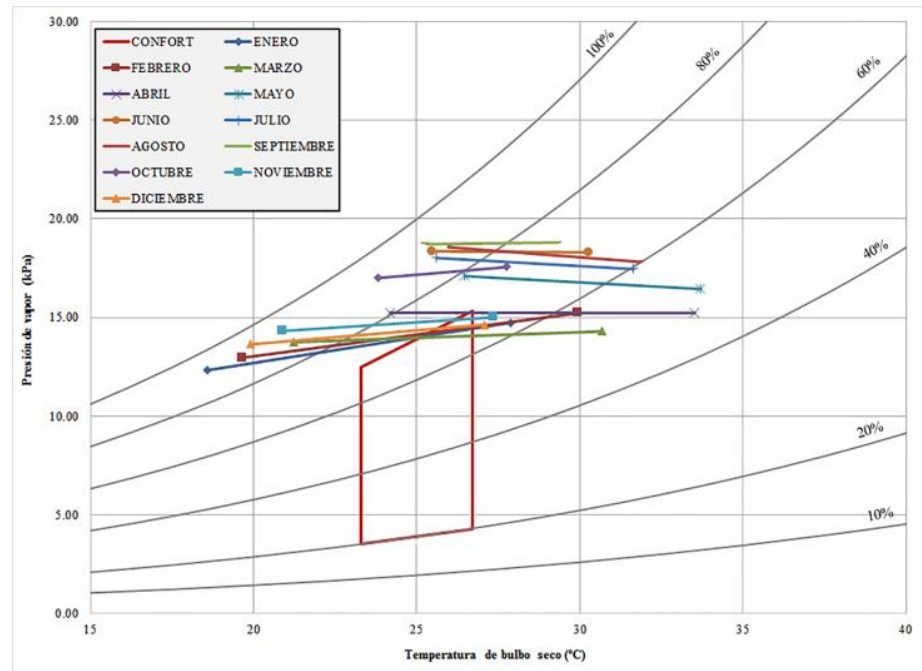

Fuente: Elaboración propia con datos de Meteoblue (2019).

Colocar los datos de esta forma en una carta bioclimática resulta beneficioso a la hora de buscar soluciones; Baruch Givoni propone una serie de recursos en su libro Man, climate and architecture (Givoni, 1969) para varias situaciones y combinaciones de temperaturas y humedades, tal como se puede observar en la figura 3. Para hacer más acertada la apreciación del confort térmico de los posibles usuarios en un sitio determinado es necesario realizar algún cálculo de sensación térmica que tome en cuenta la presión atmosférica y el viento, por lo cual se utilizó un modelo matemático publicado por Orbert G. Steadman en 1994, aunque lo hace sin tomar en cuenta la radiación solar en el balance térmico del cuerpo humano (Steadman, 1994) de la siguiente forma:

$$
\mathrm{ST}=\mathrm{Ta}+0.33 \times \mathrm{Pv}-0.70 \times \mathrm{Vv}-4.00
$$

En donde:

$\mathrm{ST}$ = Sensación térmica $\left({ }^{\circ} \mathrm{C}\right)$

$\mathrm{Ta}=$ Temperatura de bulbo seco $\left({ }^{\circ} \mathrm{C}\right)$

$\mathrm{Pv}=$ Presión del vapor de agua $(\mathrm{hPa})$

$\mathrm{V} v=$ Velocidad del viento a 10 metros de altura $(\mathrm{m} / \mathrm{s})$ 


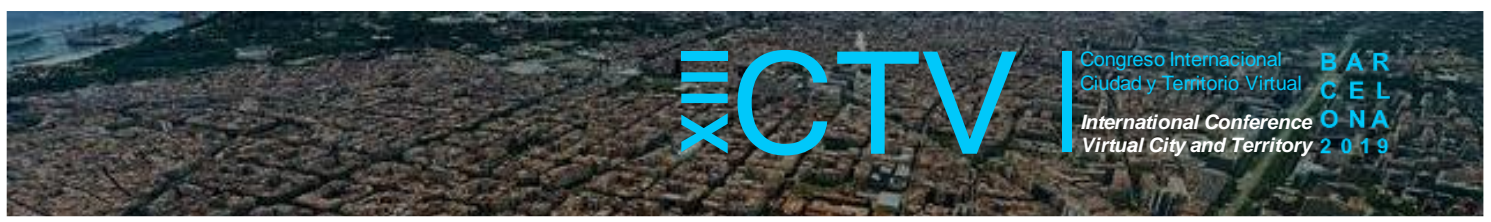

Figura 3. Estrategias bioclimáticas para lograr condiciones higrotérmicas de confort

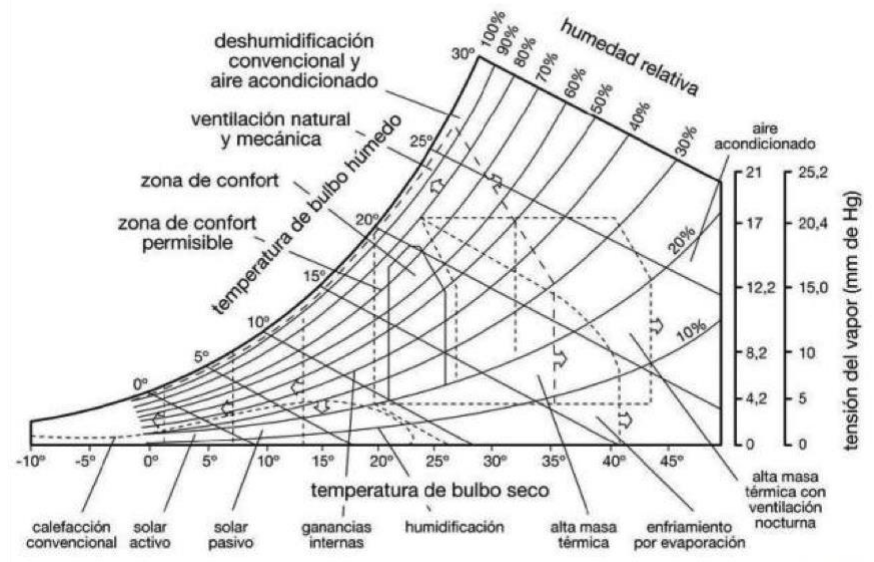

Fuente: Givoni (1969).

Dichos datos obtenidos se catalogaron de acuerdo a sus características de temperatura y humedad, como se puede observar en la figura 4, en comparación con los parámetros de confort térmico de Olgyay. Estos datos nos permiten observar que solamente se tienen condiciones climáticas de confort durante el $4.73 \%$ del año, ubicadas esencialmente en las primeras y últimas semanas del año, entre las 14:00 y las 17:00 horas. También se pueden distinguir grandes humedades en las primeras horas del día durante casi todo el año, así como una gran concentración de calor húmedo en las horas centrales desde la semana 11 y hasta la semana 38 del año. Uno de los problemas que existe en los climas tropicales es que cuando la variación de temperatura de un día es amplia, se vuelve más difícil de mantener el confort, ya que se pasa de una sensación de bochorno a la incomodidad del frío húmedo, sin puntos intermedios (Guimaraes, 2008).

Figura 4. Gráfica de calor de sensación térmica - Villahermosa, Tabasco

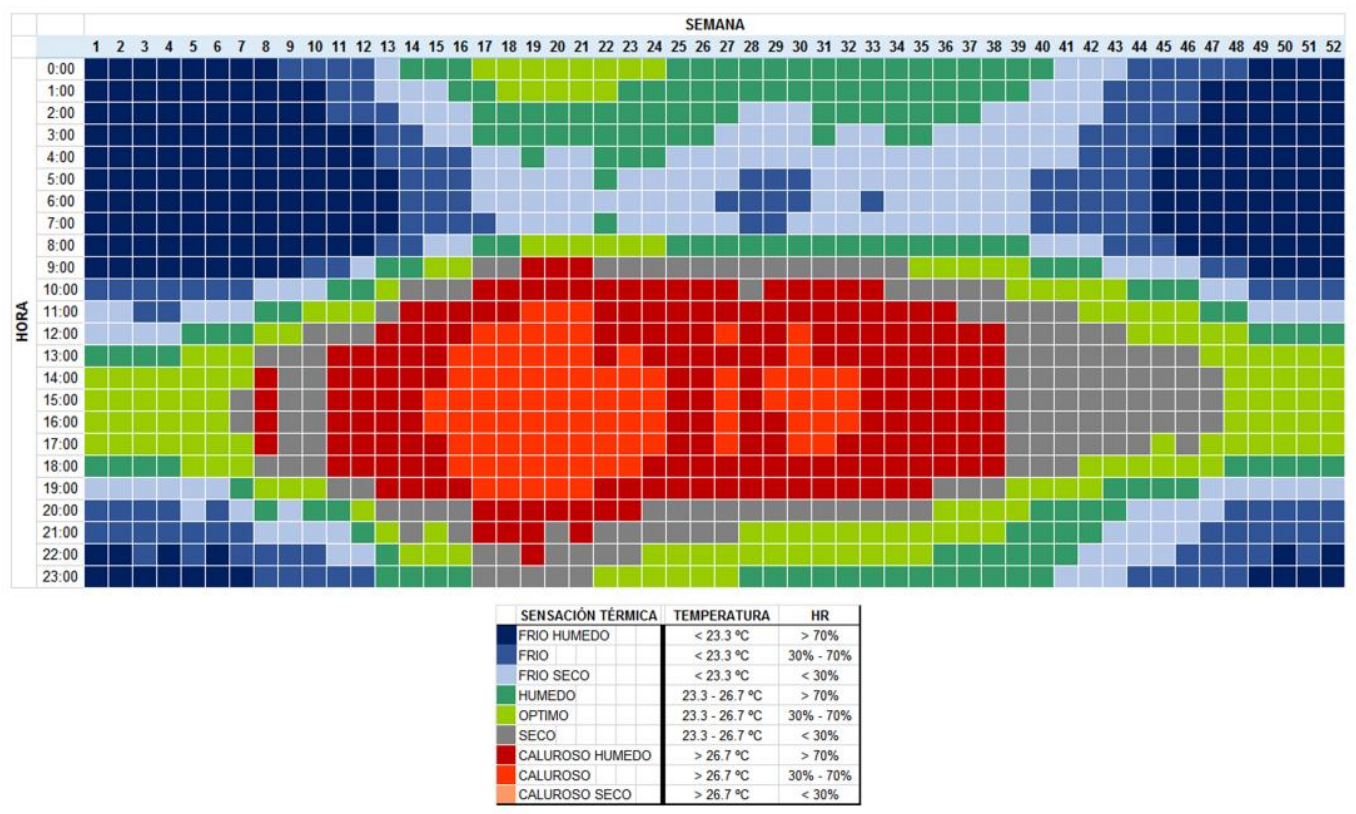

Fuente: Elaboración propia. 


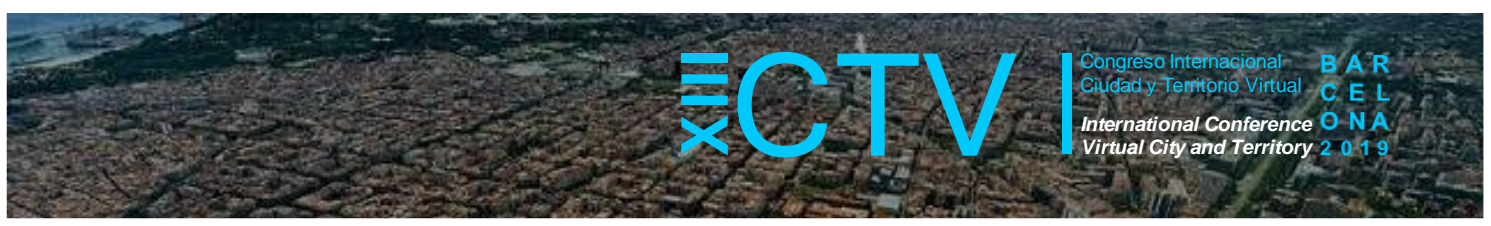

En un día promedio de la ciudad de Villahermosa, las mayores temperaturas se alcanzan entre las 14:00 y las 17:00, mientras que las temperaturas más bajas se reportan entre las 06:00 y las 07:00; entre las 20:00 y las 24:00 se mantienen temperaturas confortables, aunque la humedad relativa nunca desciende del $65 \%$, por eso en este caso se convierte en humedad absoluta para observar de manera más detallada el transcurso del día en relación a la humedad, esto se hace con la siguiente fórmula:

Y

$$
\mathrm{Ha}=622 \text { * } \mathrm{Pv} /(\mathrm{Pt}-\mathrm{Pv})
$$

$$
\mathrm{Pv}=\mathrm{Hr} / 100 \times 6.105 \times \exp ^{(17.27 \times \mathrm{Ta} /(237.7+\mathrm{Ta}))}
$$

Donde:

$\mathrm{Ha}=$ Humedad absoluta $(\mathrm{Kg})$

$\mathrm{Pv}=$ Presión de vapor de agua $(\mathrm{hPa})$

$\mathrm{Pt}=$ Presión barométrica $(\mathrm{hPa})$

$\mathrm{Hr}=$ Humedad relativa $(\%)$

$\mathrm{Ta}=$ Temperatura de bulbo seco $\left({ }^{\circ} \mathrm{C}\right)$

Los datos resultantes se agregan a la tabla 5, junto con la temperatura y entonces podemos observar que los momentos con mayor humedad en el día son entre las 09:00 y las 13:00, mientras que entre las 04:00 y las 07:00 se reportan las menores concentraciones de humedad absoluta en el ambiente. Estos datos nos permiten visualizar las horas de mayor preocupación tanto para control de humedad como de temperatura con el fin de alcanzar niveles de confort.

\section{Figura 5. Gráfica de temperatura-humedad absoluta}

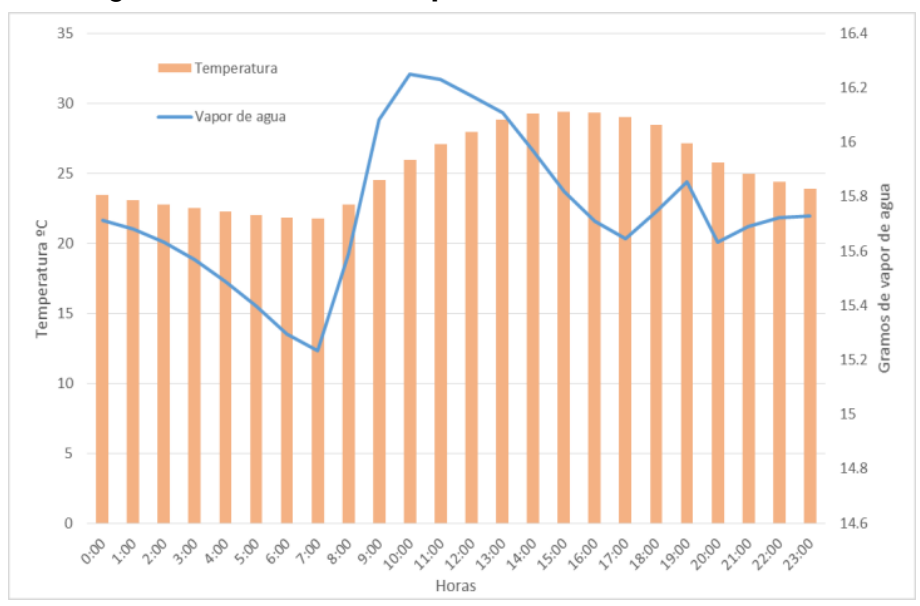

Fuente: Elaboración propia.

Tomando en cuenta todos estos valores reportados y obtenidos se define que controlar la humedad es primordial para poder lograr niveles de confort térmico. Dependiendo de los niveles de temperatura y humedad se puedes realizar muchas estrategias de diseño bioclimático, como las propuestas por Victor Olgyay o Givoni. Estas a su vez pueden ser pasivas, como ventilar, usar masa térmica, bloquear el asoleamiento, enfriar mediante evaporación, etc. O pueden ser activas, como deshumidificar, usar ventilación activa o equipos de aire acondicionado. A continuación, se analizan algunas de ellas con sus pros y contras para el caso de estudio. 


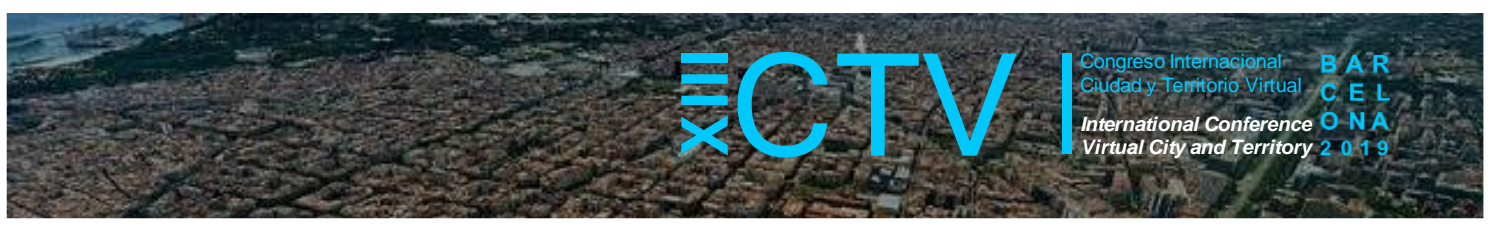

\subsection{Asoleamiento}

Para la mayoría de los días de Villahermosa, se recomienda la protección solar de las viviendas para evitar la incidencia de la radiación solar directa en el edificio, debido a que genera grandes ganancias térmicas al interior. La eficacia de dichas protecciones dependerá de muchos factores, como la cantidad de radiación, el ángulo de incidencia o la latitud de la ubicación, por lo cual es necesario que se diseñen específicamente para cada edificio y cada situación geográfica, sin embargo hay varios modelos que indican cierto coeficiente de sombra, traducido a mayor protección al interior del edificio, como se puede observar en la figura 6 , aunque también se debe de tomar en cuenta que muchas veces dichas protección imposibilitan una correcta ventilación, por lo que los sistemas móviles serían la mejor opción en el caso de estudio, un ejemplo de muestra en la figura 7.

Figura 6. Coeficiente de sombra de sistemas de protección solar

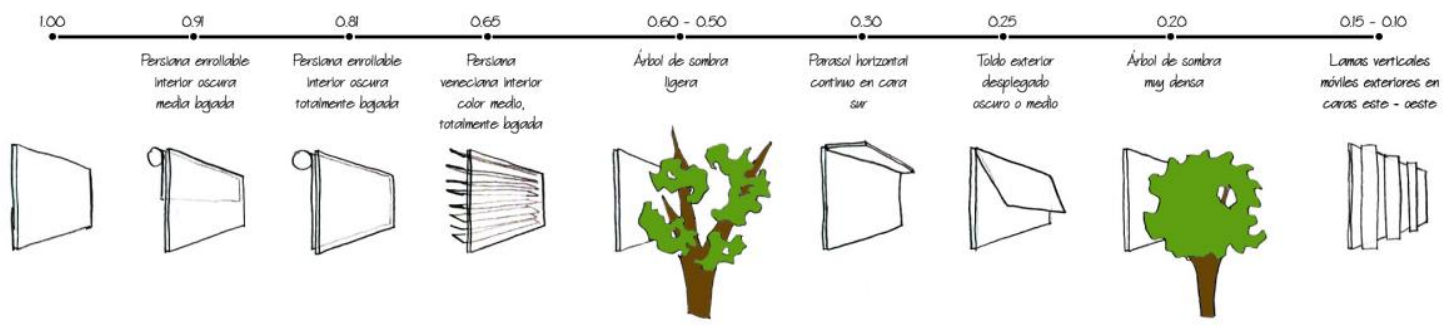

Fuente: Elaboración propia con datos de Design with Climate, Victor Olgyay, 2015.

Figura 7. Coeficiente de sombra de sistemas de protección solar

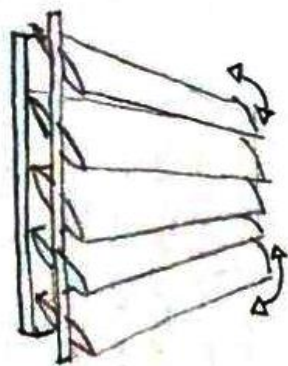

Fuente: Elaboración propia con datos de Design with Climate, Victor Olgyay, 2015.

\subsection{Ventilación y deshumidificación}

Se sugiere de igual forma el uso de ventilación natural lo más posible, renovando el aire del interior y eliminando el viciado o con exceso de humedad. Los sistemas de ventilación natural de las edificaciones son bastante populares, y pueden ser tan simples como la generación de ventilación cruzada, con dos o más aberturas situadas en fachadas opuestas, que den al exterior y de preferencia una de ellas orientada a la entrada de viento. Puede ser algo más complicado como ventilaciones de efecto chimenea como se ve en la figura 8 , sin embargo, la efectividad de estas desciende drásticamente cuando las temperaturas exteriores son muy altas, de igual forma sirve tener algún sistema automático que tome en cuenta las condiciones internas y externas para actuar. Principalmente para que la ventilación funcione como estrategia de climatización es necesario que la temperatura interior sea mayor que la temperatura exterior, de lo contrario estaríamos contribuyendo a la ganancia térmica el interior 


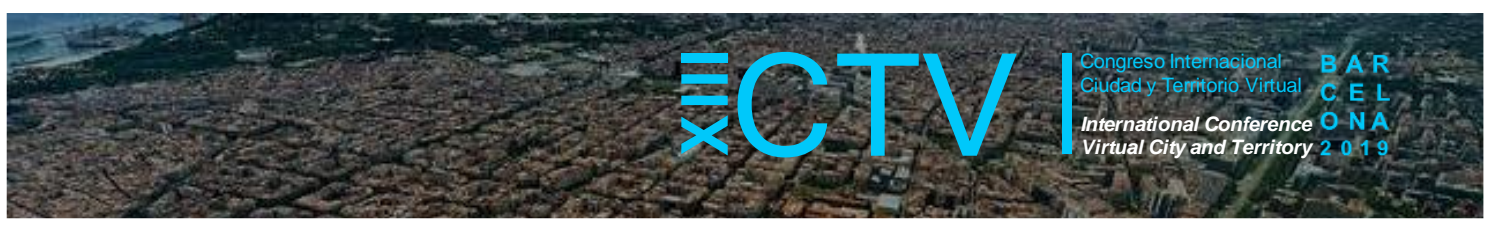

de las edificaciones, para esto es necesario tomar en cuenta el retardo que existe debido a la inercia térmica, que va en función de los materiales utilizados y espesores, así como la orientación y paso del sol.

Figura 8. Ejemplos de sistemas de ventilación natural
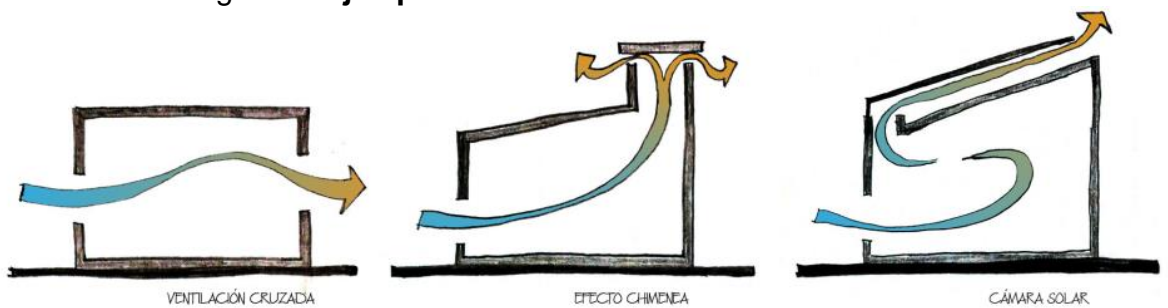

Fuente: Elaboración propia.

Para calcular la velocidad y el caudal de viento que podría entrar por una ventana orientada a la entrada de los vientos dominantes se deben de hacer algunos cálculos matemáticos propuestos por ASHRAE (1993), primeramente un ajuste por rugosidad y otro por altura real del viento:

$$
\begin{gathered}
V_{\text {ref }}=A_{\circ} * V_{\text {met }} \\
A_{0}=1.291 \exp (-0.005214(\delta-250)
\end{gathered}
$$

Donde:

$V_{\text {ref }}=$ velocidad de referencia estimada $(\mathrm{m} / \mathrm{s})$

$V_{\text {met }}=$ velocidad meteorológica $(\mathrm{m} / \mathrm{s})$

$A_{\circ}=$ constante de rugosidad

$\delta$ = capa de fricción 0 altura de la capa límite

Donde:

$$
\begin{aligned}
& \mathrm{V}_{\mathrm{H}}=\mathrm{k}{ }^{*} \mathrm{Hr}_{\mathrm{ef}}{ }^{1 / 3} \\
& \mathrm{k}=\mathrm{V}_{\mathrm{ref}} / \mathrm{H}_{\mathrm{ref}}{ }^{1 / 3}
\end{aligned}
$$

$\mathrm{V}_{\mathrm{H}}=$ velocidad del viento a la altura $\mathrm{H}(\mathrm{m} / \mathrm{s})$

$\mathrm{k}=$ constante de velocidad

$\mathrm{H}=$ altura de referencia (estación meteorológica)

Vref $=$ velocidad del viento $(\mathrm{m} / \mathrm{s})$

Posteriormente es necesario conocer la presión dinámica del viento en las ventanas para conocer después el caudal de viento que atraviesa la habitación analizada, mediante las siguientes fórmulas:

En donde:

$$
\rho w=1 / 2 p * S^{2}
$$

$\rho w=$ presión dinámica del viento $(\rho a)$

$\varsigma=$ densidad del aire ambiente -exterior $-(\mathrm{kg} / \mathrm{m} 3)$

$\mathrm{v}=$ velocidad del viento $(\mathrm{m} / \mathrm{s})$

$$
\begin{aligned}
& Q=0.827 A(\Delta \rho) 0.5 \\
& V=Q / A
\end{aligned}
$$

En donde:

$\mathrm{Q}=$ tasa de ventilación $\left(\mathrm{m}^{3} / \mathrm{s}\right)$

$A=$ área de la abertura de ventilación $\left(m^{2}\right)$

$\Delta p=$ diferencia de presiones entre las aperturas ( $\rho a)$ 


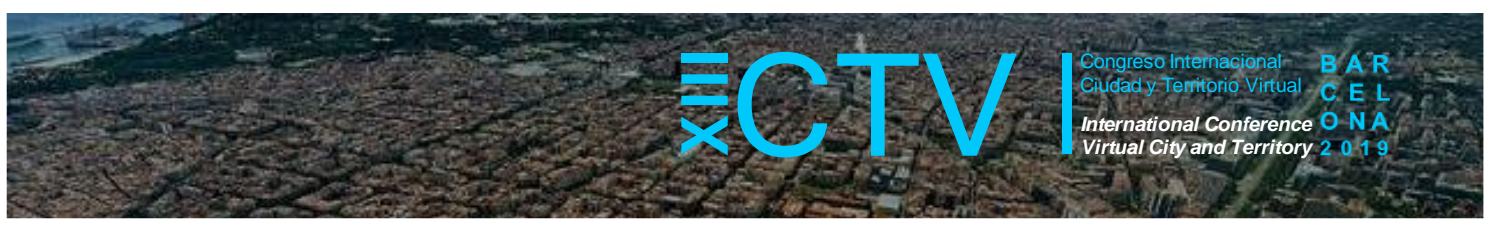

Los resultados pueden ser comparados en la tabla 2, propuesta por Evans (1957) donde se pueden ver los efectos de enfriamiento de la ventilación en el interior de las edificaciones y en la sensación térmica de sus usuarios.

Tabla 2. Velocidades del ciento en espacios interiores y su efecto en los usuarios.

\begin{tabular}{|c|c|c|c|c|c|c|c|}
\hline \multirow{3}{*}{$\begin{array}{l}\text { Vol. } \\
\text { Viento } \\
\text { (m/s) }\end{array}$} & \multirow{3}{*}{ Efecto mecánico } & \multirow{3}{*}{ Efecto en el usuario } & \multicolumn{5}{|c|}{ Efecto de enfriamiento $\mathrm{TBS}^{\circ} \mathrm{C}$} \\
\hline & & & \multicolumn{4}{|c|}{ Piel seca } & \multirow{2}{*}{$\begin{array}{c}\begin{array}{c}\text { Piel } \\
\text { húmeda }\end{array} \\
30^{\circ}\end{array}$} \\
\hline & & & $15^{\circ}$ & $20^{\circ}$ & $25^{\circ}$ & $30^{\circ}$ & \\
\hline 0.1 & $\begin{array}{l}\text { Mínimo a nivel } \\
\text { doméstico }\end{array}$ & Se puede sentir sofocación & 0 & 0 & 0 & 0 & 0 \\
\hline 0.25 & $\begin{array}{l}\text { El humo del cigarro } \\
\text { indica el movimiento }\end{array}$ & $\begin{array}{l}\text { Movimiento casi } \\
\text { imperceptible }\end{array}$ & 2 & 1.3 & 0.8 & 0.7 & 0.7 \\
\hline 0.5 & Flamear de una vela & $\begin{array}{c}\text { Se siente fresco a } \\
\text { temperaturas confortables }\end{array}$ & 4 & 2.7 & 1.7 & 1.2 & 1.2 \\
\hline 1 & $\begin{array}{l}\text { Equivale a la velocidad } \\
\text { al caminar }\end{array}$ & $\begin{array}{l}\text { Nivel máximo aceptable de } \\
\text { confort nocturno }\end{array}$ & 6.7 & 4.5 & 2.8 & 1.7 & 2.2 \\
\hline 1.5 & $\begin{array}{l}\text { Demasiado rápido para } \\
\text { trabajos de oficina. }\end{array}$ & $\begin{array}{l}\text { Límite máximo de confort } \\
\text { para actividades interiores. }\end{array}$ & 8.5 & 5.7 & 3.5 & 2 & 3.3 \\
\hline 2 & $\begin{array}{l}\text { Equivale a la velocidad } \\
\text { al caminar rápido. }\end{array}$ & $\begin{array}{c}\text { Aceptable sólo en } \\
\text { condiciones muy cálidas y } \\
\text { húmedas. }\end{array}$ & 10 & 6.7 & 4 & 2.3 & 4.2 \\
\hline
\end{tabular}

Fuente: Research report 59. Texas: texas engineering station (Evans, 1957).

Sin embargo, existe un gran problema con esta estrategia, ya que por un lado la ventilación sirve para renovar el aire interior $y$ descender las temperaturas, también resulta contraproducente en el caso de estudio, ya que introduce humedad, que ocasiona mayor incomodidad en los usuarios, sin importar si la temperatura es alta, baja o confortable. Por lo cual se deben de hacer adecuaciones pertinentes para intentar deshumidificar el aire antes de que ingrese a las edificaciones.

Una de las soluciones que se plantea es la incorporación de celdas Peltier en estructuras de aluminio colocadas en todas las ventanas de las viviendas, como en el ejemplo de la figura 9 , de tal forma que al enfriarse dichas estructuras metálicas condense el vapor de agua contenido en el aire, logrando disminuir la humedad relativa al interior. Las celdas Peltier son también conocidas como "refrigerador termoeléctrico", emplean el efecto Peltier para poder crear un flujo térmico, mediante la unión de dos tipos diferentes de materiales, generalmente aleaciones de antimonio y bismuto (DiSalvo, 1999).

Una de las principales ventajas de estos sistemas consiste en que, a diferencia de muchos otros sistemas de climatización, no tiene parte móviles, aumentando la vida útil de las celdas y disminuyendo su mantenimiento (Ghoshal, 2001). Esto convierte a este tipo de sistemas en equipos muy fiables y donde podemos predecir y simular sus resultados (Nikam \& Hole, 2014). También son considerados amigables con el medio ambiente ya que no emplean gases o algún otro medio como refrigerante, y suenen der pequeños, por lo que pueden ser aplicados en muchos lugares (Riffat \& Ma, 2003). 


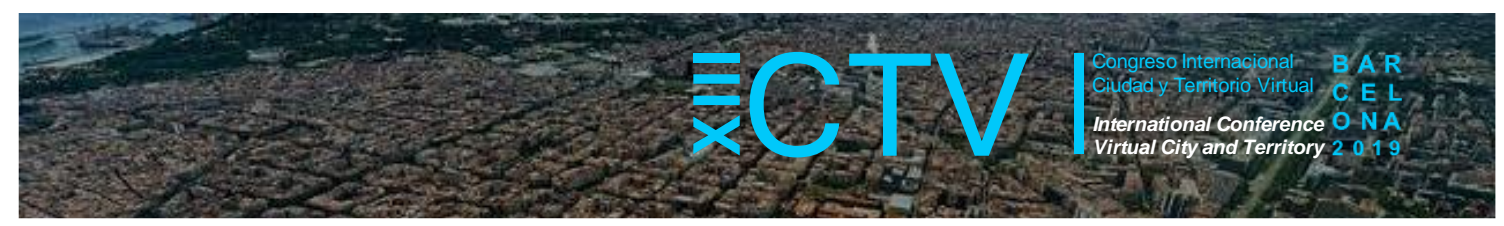

Figura 9. Ejemplos de sistemas desecantes por condensación

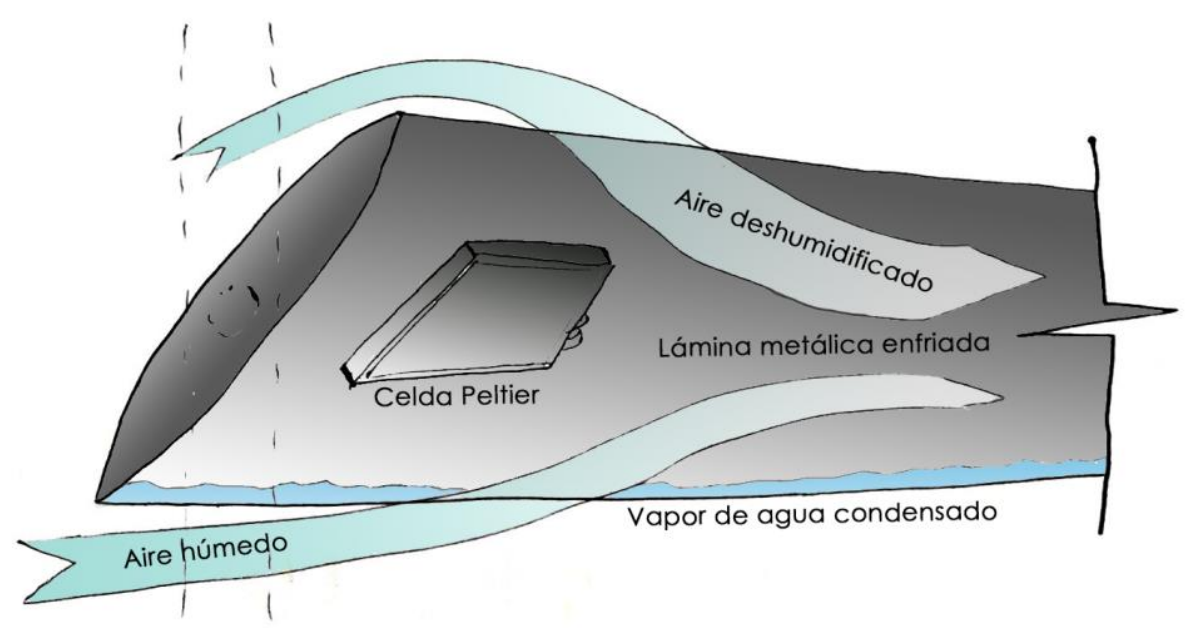

Fuente: Elaboración propia.

La deshumidificación es de suma importancia, no solo para el caso de estudio, ya que existe la tendencia reportada de humedad relativa (Coffel, Horton, \& De Sherbinin, 2017) en gran parte a que la temperatura de todo el planeta ha aumentado, y el aire caliente puede almacenar mayor cantidad de vapor de agua, por lo que se prevén problemas en sitios como el Amazonas, África central, el norte de la India y el este de China. A continuación, se presentan algunas formas de deshumidificar el ambiente.

\subsubsection{Geotermia somera pasiva y activa}

La energía geotérmica es la energía almacenada en forma de calor por debajo de la superficie de la tierra (González, 2012), por lo que es considerada una energía renovable y es utilizada ampliamente para climatizar, en especial es sitios con climas fríos (Marzolf, 2014) aunque también se ha comprobado su efectividad parcialmente como refrigerante (Andújar Márquez, Martínez Bohórquez, \& Gómez Melgar, 2016). La temperatura del suelo se mantiene constante a una profundidad de 10 metros y es prácticamente igual a la temperatura promedio del lugar (Droulia et al., 2009), que en el caso de estudio es de 25.3ㄷ. Tomando en cuenta estos factores es probable que este sistema funcione con una efectividad limitada, ya que solamente podría ser implementado cuando la temperatura al interior de la vivienda rebase los $27^{\circ} \mathrm{C}$ y pudiera existir un verdadero intercambio de temperaturas.

Sin embargo, existen modificaciones que se pueden hacer al sistema para acoplarlo de mejor forma a sitios con clima cálido-húmedo. Descender la temperatura del subsuelo en los primeros metros de profundidad es posible y ha sido comprobado con capas de grava (Derradji \& Aiche, 2014) y con riego de la superficie y bloqueo del sol (Givoni, 2007), volviendo al terreno en un potencial disipador del calor. Otra modificación posible y que se analizará con mayor profundidad es la posibilidad de implementar celdas Peltier al sistema de geotermia, con la finalidad de enfriar el aire y condensar la humedad de éste, para posteriormente reinyectar el aire a las edificaciones a menor temperatura y menos humedad. 


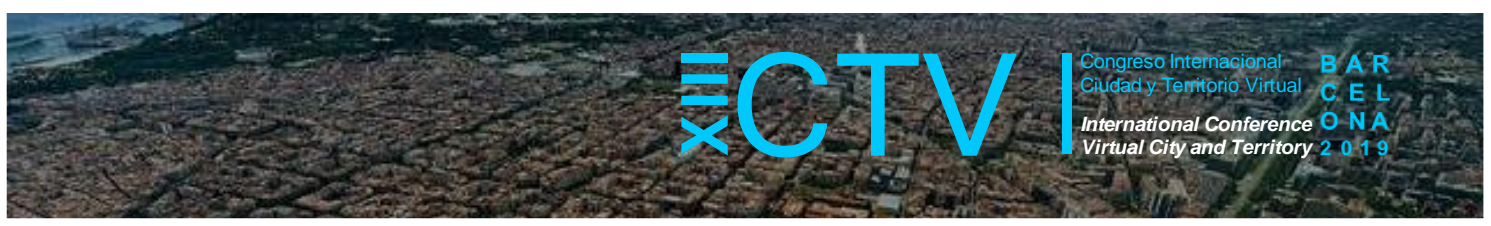

\subsubsection{Paneles desecantes}

Una de las formas más utilizadas para captar y reducir la humedad de los espacios es mediante la utilización de materiales desecantes, es decir sustancias higroscópicas que deshidratan los elementos en su proximidad, en este caso el aire. El más utilizado es el gel de sílice, debido a que no es tóxico, es inerte e insoluble en el agua (Florke et al., 2008). La capacidad de absorción de vapor de agua del gel de sílice depende de la temperatura ambiente y de los valores de presión a los que se ponga, sin embargo para el caso de estudio tiene una capacidad de absorción de $0.3 \mathrm{~kg}$ de agua por cada $\mathrm{kg}$ de sílice (Levitskij, Aristov, Tokarev, \& Parmon, 1996).

Uno de los problemas que presentan estos materiales es que cuando se saturan se requieren grandes cantidades de energía calorífica para regenerarlos. Aproximadamente se necesita calentar el gel de sílice a $120^{\circ} \mathrm{C}$ durante dos horas, cuando surge un rompimiento entre el gel de sílice y el agua contenida (Konde, 2007), sin embargo existen investigaciones donde se han empleado técnicas de bajo consumo energético para disminuir las temperaturas necesarias para dicha generación, incluso logrando la separación a los $70^{\circ} \mathrm{C}$ durante 304 horas (Y. Yao et al., 2010).

Se propone un sistema de paneles desmontables con una película de gel de sílice, que se la posibilidad de ajustarse al diseño de las viviendas, sin ser un "apéndice" que se tenga que ocultar, siendo concebido desde la proyección arquitectónica, como se ejemplifica en la figura 10.

Figura 10. Estructura de paneles desmontables deshumidificantes

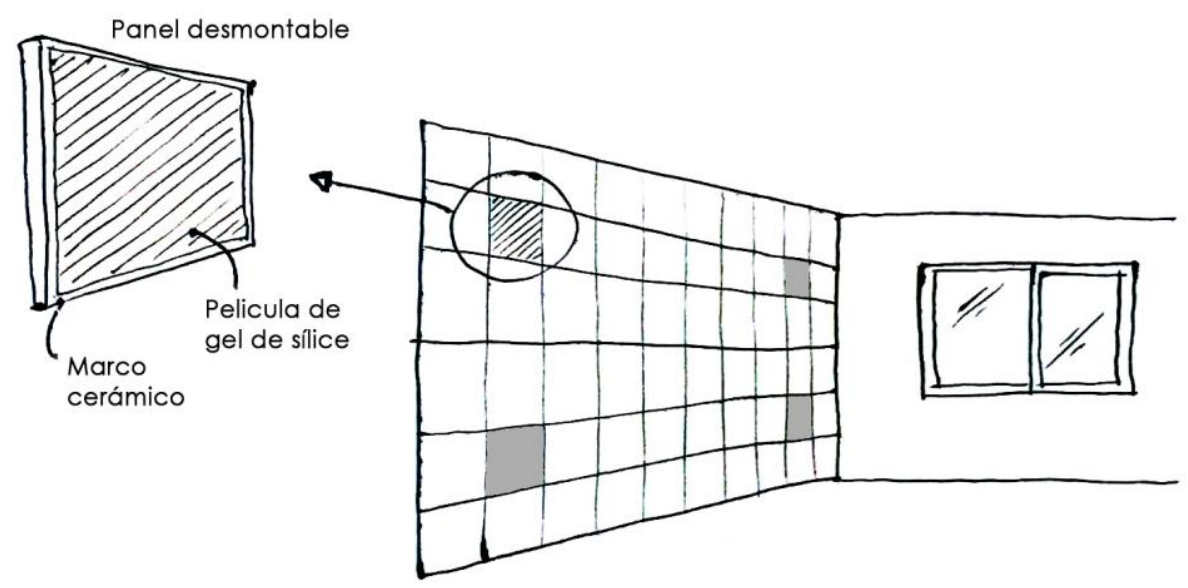

Fuente: Elaboración propia.

\subsubsection{Captación de agua de deshumidificación}

Es posible realizar un sencillo cálculo de la cantidad de agua que se podría recolectar por $\mathrm{m}^{3}$ de espacio interior, tomando en cuenta las condiciones iniciales de temperatura y humedad, así como los valores deseados después de la deshumidificación mediante la siguiente fórmula:

$$
\mathrm{m} \text { agua a remover }=\left(\omega_{\mathrm{ini}}-\omega_{\mathrm{fin}}\right) \mathrm{m} \text { aire }
$$




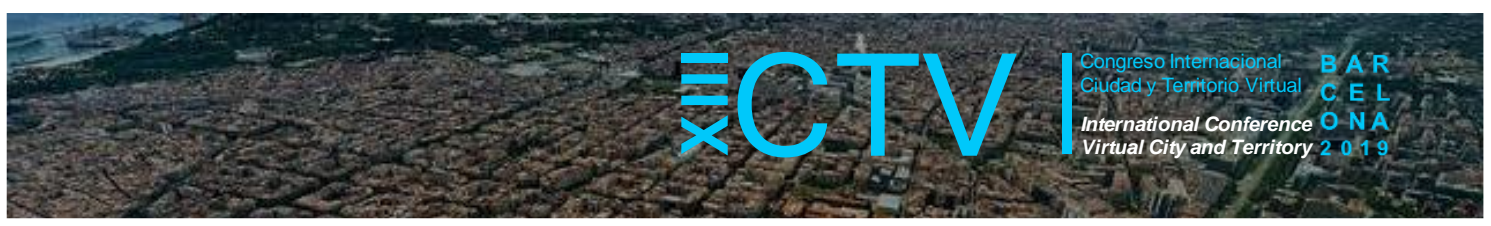

En donde:

$\mathrm{m}$ aire $=\rho$ aire * Vol (conociendo la densidad del aire promedio a nivel del mar de $1.2 \mathrm{~kg} / \mathrm{m}^{3}$ )

$\omega=$ Humedad absoluta inicial

$\%$ des = porcentaje al cual se desea reducir la humedad relativa

El resultado se deberá multiplicar por las dimensiones del lugar a deshumidificar. En caso de ser un volumen de agua muy bajo puede ser despreciado, de lo contrario puede ser reutilizado dentro de las viviendas para diversos usos.

\section{Conclusiones}

El proyecto se encuentra en una etapa inicial, en el cual se han delimitado las estrategias a emplear en el caso de estudio, siendo estas: protección solar, ventilación natural cruzada, ventilación de chimenea, deshumidificación mediante ventilación, paneles desecantes y desmontables de gel de sílice, geotermia somera activa y recuperación de agua. Es necesario realizar simulaciones y experimentación detallada para comprobar los sistemas aquí expuestos en viviendas del caso de estudio.

Existen varios problemas que impiden la fácil utilización de algunos sistemas pasivos de climatización en climas muy húmedos, por lo cual se tiene que recurrir a sistemas de bajo costo energético, de preferencia generados con energías limpias, para lograr condiciones de confort térmico. Dentro de estas alteraciones se propone la implementación de celdas Peltier por su bajo coste energético, para ayudar a los sistemas deshumidificantes, tanto en la ventilación cruzada, como en la geotermia somera.

El control de la humedad en climas cálido-húmedo es una prioridad, ya que juega un papel muy importante en la falta de confort térmico de los usuarios, por lo cual se deben de hayan soluciones económicas y ambientales para bajar dichos niveles. Posiblemente no sea posible alcanzar niveles de confort térmico durante todo el año, sin embargo la implementación de sistemas pasivos es necesaria para disminuir los altos gastos de consumo eléctrico.

Agradecimientos: Los autores quisieran agradecer al CONACYT por su apoyo.

Contribuciones de los autores: El primer autor, Ignacio Guzmán, ha desarrollado la investigación, cálculo y redacción del trabajo, bajo la supervisión y coordinación de los otros autores, Fidel Franco y Jaime Roset.

Conflicto de Intereses: Los autores declaran que no hay conflicto de intereses.

\section{Bibliografía}

Andersen, I., \& Korsgaard, J. (1986). Asthma and the indoor environment: assessment of the health implications of high indoor air humidity. Environment International, 12(1-4), 121-127.

Andújar Márquez, J. M., Martínez Bohórquez, M. Á., \& Gómez Melgar, S. (2016). Ground thermal diffusivity calculation by direct soil temperature measurement. Application to very low enthalpy geothermal energy systems. Sensors, 16(3), 306.

Arundel, A. V., Sterling, E. M., Biggin, J. H., \& Sterling, T. D. (1986). Indirect health effects of relative humidity in indoor environments. Environmental health perspectives, 65, 351-361. 




ASHRAE. (1993). Handbook Fundamentals. 1993. American Society of Heating, Refrigerating, and Air Conditioning Engineers, Atlanta.

ASHRAE. (2004). Thermal environmental conditions for human occupancy (Vol. 55): American Society of Heating, Refrigerating and Air-Conditioning Engineers.

Cervantes, J., Barradas, V., Martínez, A., Cordova, Q., Ramírez, C., \& Tepach, G. (2000). Aspectos del clima urbano de Villahermosa, Tabasco, México. Universidad y Ciencia, 16, 1016.

Coffel, E., Horton, R. M., \& De Sherbinin, A. M. (2017). Temperature and humidity based projections of a rapid rise in global heat stress exposure during the 21 st century.

Choudhury, A. R., Majumdar, P., \& Datta, C. (2011). Factors affecting comfort: human physiology and the role of clothing Improving comfort in clothing (pp. 3-60): Elsevier.

Derradji, M., \& Aiche, M. (2014). Modeling the soil surface temperature for natural cooling of buildings in hot climates. Procedia Computer Science, 32, 615-621.

DiSalvo, F. J. (1999). Thermoelectric cooling and power generation. Science, 285(5428), 703706.

Droulia, F., Lykoudis, S., Tsiros, I., Alvertos, N., Akylas, E., \& Garofalakis, I. (2009). Ground temperature estimations using simplified analytical and semi-empirical approaches. Solar Energy, 83(2), 211-219.

Evans, B. (1957). Research report 59. Texas: texas engineering station.

Florke, O. W., Martin, B., Benda, L., Paschen, S., BERGNA, H., ROBERTS, W., . . . Kleinschmit, P. (2008). Silica, Ullmann's encyclopedia of industrial chemistry: Wiley-VCH Verlag GmbH \& Co., Weinheim.

Ghoshal, U. S. (2001). Highly reliable thermoelectric cooling apparatus and method: Google Patents.

Givoni, B. (1969). Man, climate and architecture. Elsevier;().

Givoni, B. (2007). Cooled soil as a cooling source for buildings. Solar Energy, 81(3), 316-328.

González, J. P. L. (2012). Novedades y retos de la energía geotérmica en España. Paper presented at the Energía geotérmica: análisis y prospectiva.

Guimaraes, M. (2008). Confort Térmico y Tipología Arquitectónica en Clima Cálido-Húmedo: Análisis térmico de la cubierta ventilada: Universidad Politècnica de Catalunya, Barcelona, España. Recuperado el.

Hinz, E., Gonzalez, E., De Oteiza, P., \& Quiros, C. (1986). Proyecto clima y arquitectura: Universidad del Zulia. Facultad de Arquitectura. Instituto de Investigaciones de Arquitectura y Sistemas Ambientales.

INEGI. (2016). Encuesta nacional de ingresos y gastos de los hogares 2016. México. 


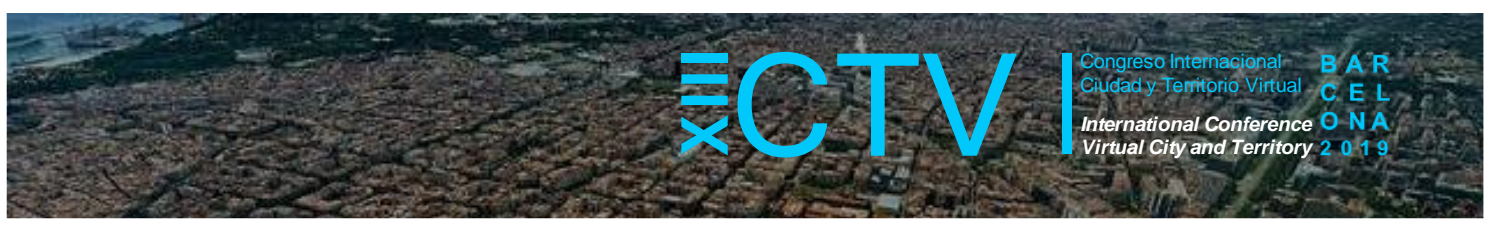

Konde, S. M. (2007). Preparation of High-Silica Zeolite Beads From Silica Gel.

Kottek, M., Grieser, J., Beck, C., Rudolf, B., \& Rubel, F. (2006). World map of the KöppenGeiger climate classification updated. Meteorologische Zeitschrift, 15(3), 259-263.

Levitskij, E., Aristov, Y. I., Tokarev, M., \& Parmon, V. (1996). "Chemical heat accumulators": a new approach to accumulating low potential heat. Solar Energy Materials and Solar Cells, 44(3), 219-235.

Marzolf, N. C. (2014). Emprendimiento de la energía geotérmica en Colombia.

Meteoblue. (2019). Tiempo en Villahermosa. Retrieved 10/09/2019, 2019, from https://www.meteoblue.com/es/tiempo/semana/villahermosa_méxico_3514670

Nikam, A. N., \& Hole, J. A. (2014). A Review on use of Peltier Effects. vol, 2, 6-12.

Olgyay, V., \& Frontado, J. (1998). Arquitectura y clima: manual de diseño bioclimático para arquitectos y urbanistas: Gustavo Gili.

Palme, M., Aldunate, C. C., \& Huerta, M. A. G. (2016). Estimación del riesgo de sobrecalentamiento y del potencial de refrigeración por ventilación natural de viviendas unifamiliares en ciudades costeras de Chile. Hábitat Sustentable, 52-61.

Riffat, S. B., \& Ma, X. (2003). Thermoelectrics: a review of present and potential applications. Applied thermal engineering, 23(8), 913-935.

Rogers, J., \& Suphachasalai, S. (2008). Residential consumption of electricity in India: documentation of data and methodology Background Paper to the India Strategies for Low Carbon Growth.

Sharmin, T., Kabir, S., \& Rahaman, M. M. (2012). A study of thermal comfort in outdoor urban spaces in respect to increasing building height in Dhaka. The AIUB Journal of Science and Engineering (AJSE), 11(1), 57-65.

Steadman, R. G. (1994). Norms of apparent temperature in Australia. Aust. Met. Mag, 43, 1-16.

Sudhakar, K., Winderl, M., \& Priya, S. S. (2019). Net-zero building designs in hot and humid climates: A state-of-art. Case Studies in Thermal Engineering, 13, 100400.

Yao, R., Li, B., \& Liu, J. (2009). A theoretical adaptive model of thermal comfort-Adaptive Predicted Mean Vote (aPMV). Building and Environment, 44(10), 2089-2096.

Yao, Y., Zhang, W., Peng, Y., Wang, L., Liu, Y., \& Chen, B. (2010). Modeling of Silica Gel Dehydration Assisted by Power Ultrasonic. 